\title{
Radtools: $\mathbf{R}$ utilities for smooth navigation of medical image data [version 1; peer review: 2 not approved]
}

\author{
Pamela H. Russell(D), Debashis Ghosh (D) \\ Biostatistics and Informatics, Colorado School of Public Health, Aurora, CO, 80045, USA
}

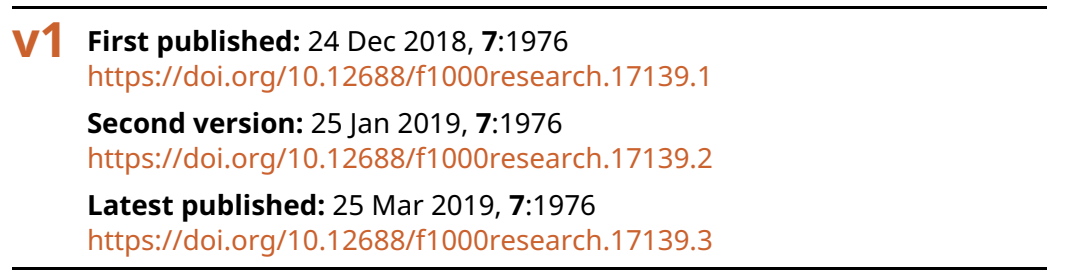

\section{Abstract}

The radiology community has adopted several widely used standards for medical image files, including the popular DICOM (Digital Imaging and Communication in Medicine) and NIfTI (Neuroimaging Informatics Technology Initiative) standards. These file formats include image intensities as well as potentially extensive metadata. The NIfTI standard specifies a particular set of header fields describing the image and minimal information about the scan. DICOM headers can include any of $>4,000$ available metadata attributes spanning a variety of topics. NIfTI files contain all slices for an image series, while DICOM files capture single slices and image series are typically organized into a directory. Each DICOM file contains metadata for the image series as well as the individual image slice.

The programming environment $\mathrm{R}$ is popular for data analysis due to its free and open code, active ecosystem of tools and users, and excellent system of contributed packages. Currently, many published radiological image analyses are performed with proprietary software or custom unpublished scripts. However, $\mathrm{R}$ is increasing in popularity in this area due to several packages for processing and analysis of image files. While these $\mathrm{R}$ packages handle image import and processing, no existing package makes image metadata conveniently accessible. Extracting image metadata, combining across slices, and converting to useful formats can be prohibitively cumbersome, especially for DICOM files.

We present radtools, an R package for smooth navigation of medical image data. Radtools makes the problem of extracting image metadata trivially simple, providing simple functions to explore and return information in familiar $\mathrm{R}$ data structures. Radtools also facilitates extraction of image data and viewing of image slices. The package is freely available under the MIT license at https://github.com/pamelarussell/radtools and is easily installable from the Comprehensive R Archive Network (https://cran.r-

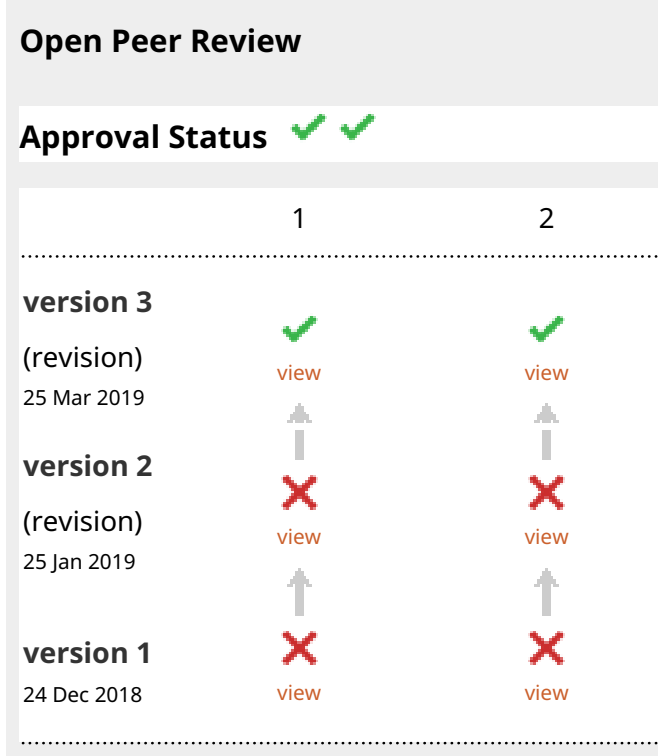

1. Andrey Fedorov ID, Harvard Medical School, Boston, USA

2. Volker Schmid (iD), Ludwig Maximilian

University of Munich, Munich, Germany

Any reports and responses or comments on the article can be found at the end of the article. 
project.org/package=radtools).

Keywords

Medical imaging, DICOM, NIfTI, R package

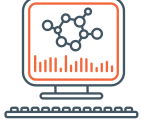

This article is included in the Bioinformatics

gateway.

This article is included in the Neuroconductor

collection.

Corresponding author: Pamela H. Russell (pamela.russell@ucdenver.edu)

Author roles: Russell PH: Conceptualization, Methodology, Software, Writing - Original Draft Preparation, Writing - Review \& Editing; Ghosh D: Conceptualization, Funding Acquisition, Writing - Review \& Editing

Competing interests: No competing interests were disclosed.

Grant information: This work has been supported by the Grohne-Stapp Endowed Chair for Cancer Research (University of Colorado Cancer Center).

The funders had no role in study design, data collection and analysis, decision to publish, or preparation of the manuscript.

Copyright: $\odot 2018$ Russell PH and Ghosh D. This is an open access article distributed under the terms of the Creative Commons Attribution License, which permits unrestricted use, distribution, and reproduction in any medium, provided the original work is properly cited.

How to cite this article: Russell PH and Ghosh D. Radtools: $\mathrm{R}$ utilities for smooth navigation of medical image data [version 1; peer review: 2 not approved] F1000Research 2018, 7:1976 https://doi.org/10.12688/f1000research.17139.1

First published: 24 Dec 2018, 7:1976 https://doi.org/10.12688/f1000research.17139.1 


\section{Introduction}

Medical image analysis often lies at the boundary of research and the clinic, presenting challenges in both domains. Institutional and privacy concerns can compete with the objective of open data for research purposes. In particular, it remains standard practice to perform analysis with proprietary software or unpublished scripts. Additionally, the majority of imaging studies do not make image data publically available due to patient privacy requirements. These complex challenges can present barriers for scientists working in the image analysis domain.

In recent years, a small but growing number of open source computational tools have been developed to process and analyze medical images, promoting sharing of code; some of the most widely adopted are described in 1-3. To address the issue of availability of public image data, our group previously developed TCIApathfinder ${ }^{4}$, an open source $\mathrm{R}$ package to simplify access to the thousands of publicly available images in The Cancer Imaging Archive ${ }^{5}$. Here, we present radtools ${ }^{6}$, an open source $\mathrm{R}$ package that lowers barriers to image analysis by simplifying the extraction of image properties and complex header information. Although several excellent image processing and analysis packages exist for the $\mathrm{R}$ environment ${ }^{2,7-10}$, none currently offers special functionality for convenient presentation of image metadata. Radtools ${ }^{6}$ implements a layer of processing to convert image metadata to familiar $\mathrm{R}$ data structures, eliminating the need for specialized knowledge and custom scripts to parse image files.

Radtools $^{6}$ supports the two most common medical image formats, DICOM (Digital Imaging and Communication in Medi-

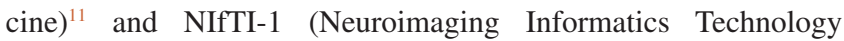
Initiative $)^{12}$. The industry standard DICOM format combines a header and two-dimensional image data into one file, so that an image acquisition typically produces multiple DICOM files. DICOM header fields consist of a "tag" that identifies the attribute, followed by the attribute value. There is no fixed size for a DICOM header; any number of thousands of possible attributes may be included. Each DICOM file for an acquisition contains its own header; many attributes will be constant across image slices. NIfTI-1 format was developed primarily for multidimensional imaging data as an improvement over the previous ANALYZE format ${ }^{12}$. NIfTI- 1 combines header information and the entire multidimensional image acquisition into either a single file or two files (one header file and one image file). Unlike DICOM, NIfTI-1 specifies a particular set of required header attributes, and the header conforms to a fixed size with an option to add extended header information. Radtools ${ }^{6}$ provides simple functions to explore and return image properties and header data from both image formats in familiar $\mathrm{R}$ data structures. Radtools ${ }^{6}$ also facilitates extraction of image data and viewing of image slices.

\section{Methods}

\section{Implementation}

Radtools $^{6}$ is provided as a package (extension to the language) for the programming language $\mathrm{R}$. The package is hosted on the Comprehensive R Archive Network (CRAN), and can be installed into the user's local $\mathrm{R}$ environment with the command 'install. packages(“radtools")'. The package is loaded into an R session or script with the command 'library(radtools)'. Radtools consists of a collection of functions that can be called within $\mathrm{R}$ scripts or interactively from the $\mathrm{R}$ console. Package usage is documented in a vignette that can be viewed on the GitHub page (https:// github.com/pamelarussell/radtools), the CRAN page (https:// cran.r-project.org/package=radtools), or from the $\mathrm{R}$ console with the command 'browseVignettes("radtools")'. The package reference manual provides documentation of each individual function and is available on the CRAN page.

\section{Operation}

The only system requirement is a working installation of $\mathrm{R}$ version $\geq 3.4$.0. The radtools workflow consists of calling radtools functions from the $\mathrm{R}$ console or within $\mathrm{R}$ scripts.

\section{Use cases}

Radtools $^{6}$ can extract image properties and header data from any valid DICOM or NIfTI-1 file. Image datasets are loaded with the 'read_dicom' and 'read_nifti1' functions. Several generic functions extract attributes from either data type, including ‘img_dimensions`, ‘num_slices`, ‘header_fields', which reports the set of header fields present, and 'header_value', which returns the value(s) of a particular attribute. Additionally, functions are provided to specifically address one format or the other. All header data present in a DICOM acquisition can be extracted into a matrix, where rows are attributes and columns are slices, with the 'dicom_metadata_matrix' function. As most DICOM headers contain numerous attributes and many of these are constant across all slices, the 'dicom_constant_header_values' function produces a named list of common attributes across slices. NIfTI-specific functions include 'nifti1_num_dim`, which returns the number of dimensions, and 'nifti1_header_values', which returns a named list of all metadata attributes for the image.

The image itself can be extracted as a multidimensional matrix of intensities for either file format with 'img_data_to_mat'. Image slices can be visualized with `view_slice`.

Finally, functions are provided to explore aspects of the DICOM standard itself. The functions 'dicom_all_valid_header_tags', 'dicom_all_valid_header_names', and 'dicom_all_valid_header_ keywords` return complete lists of valid DICOM header attributes. The functions `dicom_search_header_names' and 'dicom_search_ header_keywords`return attributes matching a search term.

\section{Conclusions}

Radtools $^{6}$ fills a specific need in the existing ecosystem of $\mathrm{R}$ packages for image processing and analysis: namely, the need for smooth extraction of image metadata. The package will accelerate workflow development and provide researchers with easy access to attributes that they may not have otherwise considered using. The inclusion of the package on CRAN, along with clear documentation, make it trivially simple for $\mathrm{R}$ users to obtain and begin using radtools.

\section{Data availability}

No data are associated with this article. 


\section{Software availability}

Radtools can be installed with the $\mathrm{R}$ command "install. packages("radtools").

Radtools is available from CRAN: https://cran.r-project.org/ package $=$ radtools.

Source code available from: https://github.com/pamelarussell/ radtools.

Archived source code at time of publication: http://dx.doi. org/10.5281/zenodo. $1477093^{6}$.
License: MIT License.

Grant information

This work has been supported by the Grohne-Stapp Endowed Chair for Cancer Research (University of Colorado Cancer Center).

The funders had no role in study design, data collection and analysis, decision to publish, or preparation of the manuscript.
1. van Griethuysen JJM, Fedorov A, Parmar C, et al.: Computational Radiomics System to Decode the Radiographic Phenotype. Cancer Res. 2017; 77(21): e104-7. PubMed Abstract | Publisher Full Text | Free Full Text

2. Whitcher B, Schmid V, Thorton A: Working with the DICOM and NIfTI Data Standards in R. J Stat Softw. Articles. 2011; 44(6): 1-29. Publisher Full Text

3. Fedorov A, Beichel R, Kalpathy-Cramer J, et al.: 3D Slicer as an image computing platform for the Quantitative Imaging Network. Magn Reson Imaging. 2012; 30(9): 1323-41.

PubMed Abstract | Publisher Full Text | Free Full Text

4. Russell P, Fountain K, Wolverton D, et al: TCIApathfinder: An R Client for the Cancer Imaging Archive REST API. Cancer Res. 2018; 78(15): 4424-6. PubMed Abstract | Publisher Full Text

5. Clark K, Vendt B, Smith K, et al.: The Cancer Imaging Archive (TCIA): maintaining and operating a public information repository. J Digit Imaging. 2013; 26(6): 1045-57.

PubMed Abstract | Publisher Full Text | Free Full Text

6. Russell P: pamelarussell/radtools: 1.0.1 (Version v1.0.1). Zenodo. 2018. http://www.doi.org/10.5281/zenodo.1477093

7. Get Images Out of DICOM Format Quickly. [R package divest version 0.7.1] [cited 2018 Nov 6]. Reference Source

8. Clayden JD, Maniega SM, Storkey AJ, et al:: TractoR: Magnetic Resonance Imaging and Tractography with R. J Stat Softw. Articles. 2011; 44(8): 1-18. Publisher Full Text

9. Fast R and $C_{++}$Access to NIfTI Images [R package RNifti version 0.10.0]. [cited 2018 Nov 6] Reference Source

10. Bordier C, Dojat M, Micheaux P: Temporal and Spatial Independent Component Analysis for fMRI Data Sets Embedded in the AnalyzeFMRI R Package. J Stat Softw. Articles. 2011; 44(9): 1-24. Publisher Full Text

11. DICOM Standard. [cited 2018 Nov 5] Reference Source

12. Jenkinson M: NIfTI-1 Data Format - Neuroimaging Informatics Technology Initiative. 2005. [cited 2018 Nov 5]

Reference Source

13. FormatAnalyze - MRC CBU Imaging Wiki. [cited 2018 Nov 6].

Reference Source 


\section{Open Peer Review}

\section{Current Peer Review Status: $\mathrm{X} \times$}

\section{Version 1}

Reviewer Report 07 January 2019

https://doi.org/10.5256/f1000research.18738.r42220

(C) 2019 Schmid V. This is an open access peer review report distributed under the terms of the Creative Commons Attribution License, which permits unrestricted use, distribution, and reproduction in any medium, provided the original work is properly cited.

\section{Volker Schmid}

Department of Statistics, Ludwig Maximilian University of Munich, Munich, Germany

This manuscript describes the $\mathrm{R}$ package radtools. The aim of the $\mathrm{R}$ package is "smooth navigation of medical image data".

The idea to provide functions which appear "smooth" for the end user is of great importance. However, the functions provides in the package do not seem to be of much (additional) benefit to the end user. Functions for reading NIfTI and DICOM images are wrappers around function in the packages oro.nifti and oro.dicom; visualisations of medical images are realised in oro.nifti. Only the functions for exploring DICOM headers are genuinely original. This is of course an important part of working with (DICOM) images.

From my understanding, F1000Research requires software tools articles to contain examples of the use of the tools. This manuscript does not contain any examples. An example (or two examples) would not only strengthen the manuscript, but could/would also show the benefit of the R package itself.

Is the rationale for developing the new software tool clearly explained?

No

Is the description of the software tool technically sound? Partly

Are sufficient details of the code, methods and analysis (if applicable) provided to allow replication of the software development and its use by others?

Yes

Is sufficient information provided to allow interpretation of the expected output datasets and any results generated using the tool? 
No

Are the conclusions about the tool and its performance adequately supported by the findings presented in the article?

No

Competing Interests: I am the co-author of the R packages oro.dicom and oro.nifti, which have been used and cited by the authors (however, I am currently not actively developing either package)

Reviewer Expertise: statistical analysis of medical images

I confirm that I have read this submission and believe that I have an appropriate level of expertise to state that I do not consider it to be of an acceptable scientific standard, for reasons outlined above.

Reviewer Report 07 January 2019

https://doi.org/10.5256/f1000research.18738.r42221

(c) 2019 Fedorov A. This is an open access peer review report distributed under the terms of the Creative Commons Attribution License, which permits unrestricted use, distribution, and reproduction in any medium, provided the original work is properly cited.

\section{Andrey Fedorov}

Department of Radiology, Brigham and Women's Hospital, Harvard Medical School, Boston, MA, USA

The authors present a new R package developed to support the use of DICOM and NIfTI files from the $\mathrm{R}$ environment. The authors rightfully discuss the popularity of $\mathrm{R}$ and the need to support image processing tasks in this environment. The argument for development of the proposed package, radtools, is that "[...] no existing package makes image metadata conveniently accessible. Extracting image metadata, combining across slices, and converting to useful formats can be prohibitively cumbersome, especially for DICOM". The resulting package is available from CRAN, and this reviewer confirmed its installation and basic functions.

The major issues that need to be addressed to make the article sound are the following:

1. Justification of the development of a new package for working with DICOM, or with NIfTI, is not sufficient.

2. No details are provided about how the functionality was tested, and about the capabilities and limitations of the package in terms of supporting specific DICOM objects.

3. Related to 2), no details are provided about how the DICOM files are handled "under the hood", i.e., whether all IO functionality was implemented from scratch, or the package is using some other DICOM libraries.

Through the text, the authors reference other R packages for similar tasks, and most notably oro.dicom and oro.nifti ${ }^{1}$. Those packages have been around for quite a long time, are broadly 
used, based on citations of the corresponding articles, and arguably provide the functionality of the proposed new package (loading data in the aforementioned formats, examination of the attributes, visualization of the images), plus more (e.g., writing of the NIfTI data).

DICOM is a complex standard, with a lot of ways information can be stored. For example, there are different methods to encode the content (transfer syntax), different character sets that can be used, private attributes. Therefore, often the quality of a DICOM implementation is defined to a large degree by the data that was used to test the implementation. The quality is also usually improved over time with the usage of the implementation. The proposed package is not accompanied by any details about what types of DICOM objects are supported, what was tested and how. Given it is a new package with a short development and usage history, one has to make a very strong argument for introducing such new tools in presence of existing alternatives.

Other suggestions:

The discussion of the DICOM objects is an oversimplification, which is reflected in the implementation of the functionality. The standard defines various types of objects that can be serialized as files, but those objects are not limited to images. As an example, DICOM defines Structured Reporting object, which will not have PixelData. The proposed package fails to read such object. The authors can find sample SR objects in the familiar to them TCIA (e.g., see QIN-HEADNECK collection).

"smooth" is a subjective qualifier that is redundant in the title and the text.

I will be happy to reconsider this article after the authors address the above concerns. But my current opinion is that oro.dicom and oro.nifti set a rather high bar for any new implementation of similar functionality.

\section{References}

1. Whitcher B, Schmid V, Thornton A: Working with the DICOM and NIfTI Data Standards inR. Journal of Statistical Software. 2011; 44 (6). Publisher Full Text

Is the rationale for developing the new software tool clearly explained?

No

Is the description of the software tool technically sound?

No

Are sufficient details of the code, methods and analysis (if applicable) provided to allow replication of the software development and its use by others?

Yes

Is sufficient information provided to allow interpretation of the expected output datasets and any results generated using the tool?

Yes

Are the conclusions about the tool and its performance adequately supported by the findings presented in the article? 
No

Competing Interests: No competing interests were disclosed.

Reviewer Expertise: medical image computing, imaging informatics, applications of DICOM for implementing FAIR principles in medical image computing

I confirm that I have read this submission and believe that I have an appropriate level of expertise to state that I do not consider it to be of an acceptable scientific standard, for reasons outlined above.

The benefits of publishing with F1000Research:

- Your article is published within days, with no editorial bias

- You can publish traditional articles, null/negative results, case reports, data notes and more

- The peer review process is transparent and collaborative

- Your article is indexed in PubMed after passing peer review

- Dedicated customer support at every stage

For pre-submission enquiries, contact research@f1000.com

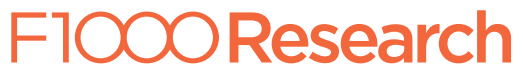

